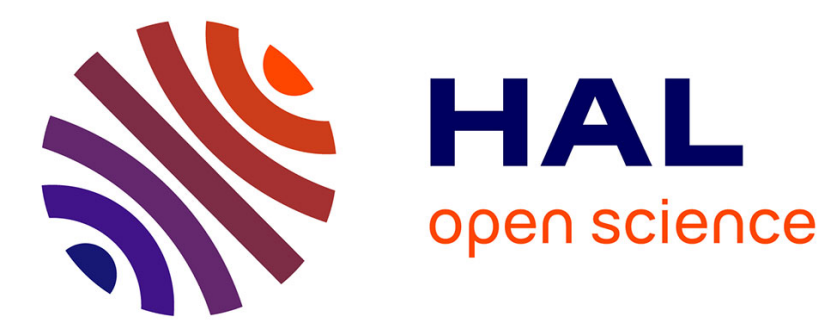

\title{
Collective Resonances of a Chain of Coupled Phononic Microresonators
}

Tingting Wang, Sylwester Bargiel, Franck Lardet-Vieudrin, Yan-Feng Wang, Yue - Sheng Wang, Vincent Laude

\section{- To cite this version:}

Tingting Wang, Sylwester Bargiel, Franck Lardet-Vieudrin, Yan-Feng Wang, Yue - Sheng Wang, et al. Collective Resonances of a Chain of Coupled Phononic Microresonators. Physical Review Applied, 2020, 13 (1), pp.014022 (10). hal-02993967

\section{HAL Id: hal-02993967 \\ https://hal.science/hal-02993967}

Submitted on 7 Nov 2020

HAL is a multi-disciplinary open access archive for the deposit and dissemination of scientific research documents, whether they are published or not. The documents may come from teaching and research institutions in France or abroad, or from public or private research centers.
L'archive ouverte pluridisciplinaire $\mathbf{H A L}$, est destinée au dépôt et à la diffusion de documents scientifiques de niveau recherche, publiés ou non, émanant des établissements d'enseignement et de recherche français ou étrangers, des laboratoires publics ou privés. 


\title{
Collective resonances of a chain of coupled phononic micro-resonators
}

\author{
Ting-Ting Wang, ${ }^{1,2}$ Sylwester Bargiel, ${ }^{2}$ Franck Lardet-Vieudrin, ${ }^{2}$ \\ Yan-Feng Wang, ${ }^{3, *}$ Yue-Sheng Wang, ${ }^{1,3}$ and Vincent Laude ${ }^{2, \dagger}$ \\ ${ }^{1}$ Institute of Engineering Mechanics, Beijing Jiaotong University, 100044 Beijing, China \\ ${ }^{2}$ Institut FEMTO-ST, Université Bourgogne Franche-Comté and CNRS, 25030 Besançon, France \\ ${ }^{3}$ School of Mechanical Engineering, Tianjin University, 300350 Tianjin, China
}

\begin{abstract}
We study experimentally a chain of defect resonators in a phononic crystal slab and observe its collective resonances at ultrasonic frequencies of a few MHz. A phononic crystal of cross-holes is fabricated in a thin fused silica plate by femtosecond laser writing followed by $\mathrm{KOH}$ etching. A chain of 17 coupled resonators is defined with no definite spatial periodicity but similar coupling strength between nearest neighbor resonators. The full phononic band gap ensures that there are only evanescent waves in the crystal which can tunnel between adjacent resonators in the plane. The resulting evanescent coupling strength decreases exponentially with distance. Collective resonances are excited by a frequency-driven piezoelectric vibrator attached at one end of the chain and imaged along the chain with a laser Doppler vibrometer. A discrete spectrum of resonances is observed and explained by a model representing the chain as a phononic polymer. The theoretical analysis is supported by finite element simulations that agree well with experimental results. Chains of evanescently-coupled micro-resonators forming phononic polymers could find applications for ultrasonic sensing, for implementation in topological phononics, and for the design of optomechanical resonator chains.
\end{abstract}

\section{INTRODUCTION}

Over the past two decades, a great deal of effort has been made to achieve control of waves propagating within phononic/sonic crystals exhibiting elastic/acoustic band gaps $^{1}$. The propagation of waves is completely forbidden in band gaps ${ }^{2}$. Actually, even though there are no waves propagating inside a band gap of a perfect crystal, evanescent waves still exist ${ }^{3}$. Besides explaining complex solutions in the dispersion relation of homogeneous media and crystals ${ }^{4}$, evanescent waves also provide a coupling mechanism for the vibrations of periodic chains of masses connected by spring ${ }^{5,6}$ or of coupled-resonator waveguides $^{7,8}$. Strong confinement and localization of waves can indeed be achieved at the frequencies in the complete phononic band gap. ${ }^{9-11}$. When the periodicity of the perfect crystal is broken, for instance by changing the geometry of the unit cell or the material properties, point and line defects can form cavities and waveguides, respectively ${ }^{12,13}$.

Waveguides composed of linear chains of coupled resonators in a crystal were first proposed in the field of photonics ${ }^{14}$. These coupled-resonator waveguides can be designed using photonic-crystal defect cavities or micro-ring resonators ${ }^{15}$, and can have applications to slow light ${ }^{16}$, light storage $^{17}$, sensing ${ }^{18}$, and light capture ${ }^{19}$. Dielectric coupled-resonator waveguides also parallel plasmonic waveguides composed of periodically distributed metal nanoparticules ${ }^{20}$. Coupled-resonator waveguides have been since then extended to the field of phononic crystals. Khelif et al. demonstrated experimentally the guiding and the bending of acoustic waves in highly confined waveguides ${ }^{21}$. Hatanaka et al. developed a phonon waveguide using a one-dimensional array of suspended membranes and demonstrated that it could support and guide mechanical vibrations by nano-electro-mechanical systems (NEMS $)^{22}$. Wang et al. investigated experimentally Lamb wave propagation in coupled-resonator elastic waveguides formed by a chain of cavities in a two-dimensional phononic crystal slab with cross holes ${ }^{23}$. They also investigated the transmission properties of coupled-resonator acousto-elastic waveguides formed by a chain of cavities with water, such as multiply $90^{\circ}$-bent waveguides or wave splitters ${ }^{24}$.

Defects in a phononic crystal have long been known to introduce localized states of vibration ${ }^{25}$. They have been proposed to filter the transmission through a crystal sample ${ }^{26}$ or to alter the transmission along a waveguide $^{27}$. In general, the defects used were small and chosen to possess well defined isolated resonance frequencies. An additional condition of periodicity at the level of a super-cell is generally imposed, in order to guarantee that the dispersion can be obtained numerically via the computation of phononic band structures. In this paper, we instead examine an extended and non periodic defect in a phononic crystal, basing on the coupled-resonator concept, that possesses a discrete spectrum of closelyspaced resonances. We remark that coupling between adjacent resonators is not limited to straight lines or to privileged crystallographic directions. Instead, evanescent coupling inside a complete band gap is omnidirectional and decreases exponentially away from a resonator. Hence, it provides an adequate platform to implement nearest-neighbor coupling within an aperiodic chain of finite length, without the additional propagation phases that would result from attaching the resonators to a propagation medium or a surface ${ }^{28,29}$. Actually, interaction between adjacent resonators is reminiscent of the chemical bonds of molecular polymers. Thus the chain of resonators is loosely termed a 'phononic polymer' in this work and it is understood that it can be deformed rather freely in the plane. 
(a)

step 1: 3D design of laser pattern step 2: Direct laser writing

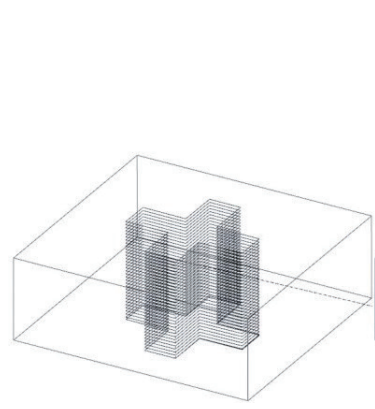

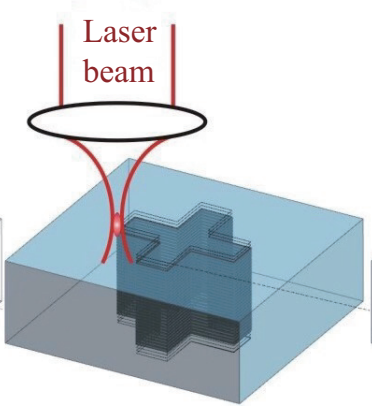

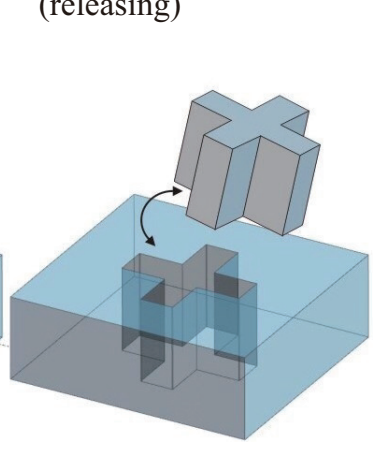

step 3: Wet etching (releasing)

(c)

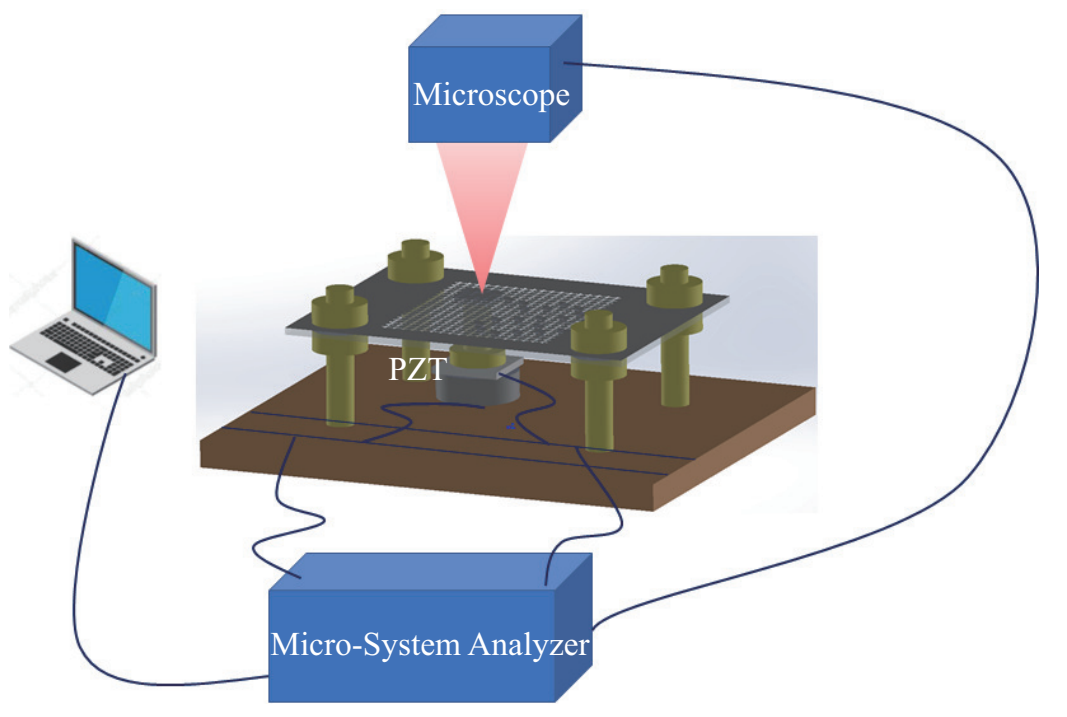

(b)

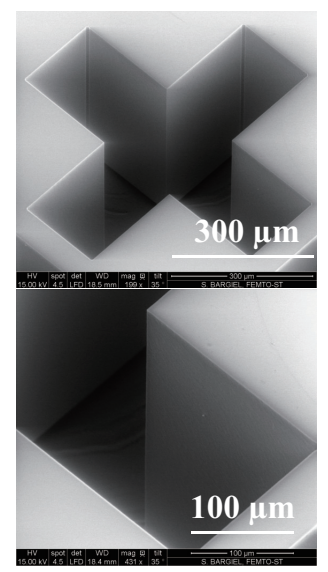

(d)

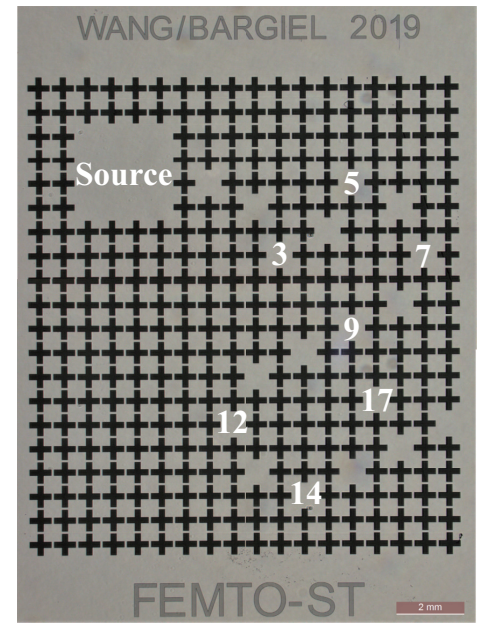

Figure 1. Experimental setup for excitation and imaging of the vibrations of a chain of coupled resonators in a phononic crystal slab. (a) A array of cross holes forming a square lattice is etched in a fused silica plate by femtosecond laser-assisted wet etching. (b) A scanning electron microscope image of a single cross hole and a zoomed view at a sidewall, illustrating the fabrication roughness, are shown. (c) A sketch of the experimental setup shows the fused silica sample attached to a PCB by screws and nuts. The bottom surface is in contact with a piezoelectric patch (PZT) acting as a source of vibrations. Out-of-plane vibrations are detected at the top surface with a laser micro-system analyzer. (d) An optical microscope image of the sample shows the chain of 17 coupled resonators embedded in a $20 \times 18$ phononic crystal of cross holes and the position of the source of vibrations.

In the following, we specifically consider a chain of coupled resonators fabricated in a phononic crystal slab in fused silica that can be conveniently manufactured at the microscale. The phononic crystal slab of cross holes possesses a wide complete band gap extending from 1.5 to $4 \mathrm{MHz}$. The chain is formed by distributing resonators along an aperiodic path but respecting an equal coupling scheme. Collective modes of vibration of the chain are imaged thanks to a laser vibrometer. A fine spectrum of resonances involving the motion of all resonators at once is observed. Finally, a theoretical model is proposed to explain the frequency response and to predict the discrete spectrum of resonance frequencies.

\section{METHODS}

Recently, femtosecond laser-assisted wet etching has been demonstrated as a powerful mask-free micromachining method, allowing fabrication of various 3D microstructures in fused silica $\left(\mathrm{a}-\mathrm{SiO}_{2}\right)$ and other glass materials with high spatial precision ${ }^{30}$. This method is well suited for the fabrication of phononic crystals resonators due to the high selectivity of etching of glass materials in $\mathrm{KOH}(>200)$, resulting in high aspect ratio structures with well-defined and smooth vertical sidewalls. In this work, the process parameters were adjusted to produce a significant increase in the etch rate of irradiated silica ( $>200$ times in $\mathrm{KOH}$ ). Increased etch rate can be 
explained by laser-induced stress generation that is responsible for weakened chemical bonds (and hence bond angle variations) in the $\mathrm{SiO}_{2}$ matrix ${ }^{31}$. Moreover, with increasing number of laser pulses, stress builds up and a porous structure is created that can be penetrated much faster by the etchant.

The chain of coupled resonators shown in Fig. 1 was manufactured in a fused silica plate. The sample size is $26 \mathrm{~mm} \times 20 \mathrm{~mm} \times 500 \mu \mathrm{m}$. A square lattice of cross holes, with lattice constant $a=714 \mu \mathrm{m}$, was etched in the plate by using a 3D laser system (FEMTOprint model f100 aHEAD Enhanced), equipped with a $5 \mathrm{~W}$ femtosecond laser operating at $\lambda=1030 \mathrm{~nm}$. The overall fabrication process is a direct writing method (i.e., without mask) and is composed of three main steps. The process flow is shown in Figure 1(a). First, a laser machining process is programmed in $3 \mathrm{D}$ by use of a dedicated software (Alphacam) that results in the generation of a machining code file. Next, the fused silica substrate is scanned by the focused laser beam, according to the program, and the material is exposed to low-energy femtosecond pulses that accelerate locally the etching rate. Only a very thin contour (2-2.5 $\mu \mathrm{m}$ wide) of each cross-hole is exposed through the whole substrate thickness in sequential order. In the final step, the thin walls of exposed silica (aspect ratio $\approx 80$ ) are double-side etched in a $10 \mathrm{M} \mathrm{KOH}$ solution at $80^{\circ} \mathrm{C}$. The total etching time was $3 \mathrm{~h}$ (etch rate $\approx 2.2 \mu \mathrm{m} / \mathrm{min}$ ) resulting in releasing of silica cross blocks into the etchant. Ultrasonic vibrations were activated periodically during etching to enhance penetration of $\mathrm{KOH}$ through the high aspect structure and to stabilize the etching rate. The scanning electron microscope image of a cross hole (measured with SEM Quanta 450W) in Fig. 1(b) allows to verify the smoothness of the walls after etching. Fabrication errors are estimated to be less than $\pm 1 \mu \mathrm{m}$.

Figure 1(c) shows a sketch of the experimental setup. The sample is held over a printed circuit board (PCB) by using screws and nuts. Its bottom surface is in contact with a piezoelectric ceramic transducer (PZT) that acts as a source of vibrations. We use a MEGGIT Pz27 (Navy II) soft relaxor type PZT whose fundamental resonant frequency is $5 \mathrm{MHz}$ to cover the whole frequency range of interest. Pz27 is an all-round soft PZT material with good coupling factor, good charge coefficients, high Curie temperature, and low mechanical quality factor. We avoid resonance effects in the transducer by working below its fundamental resonance frequency. The transformed vertical displacement vibration signal is transmitted to the sample through a contacting screw.

We designed the chain of resonators by omitting the etching of selected holes, as shown in Fig. 1(d). We purposely chose the sequence of defects to follow a rather arbitrary path in the plane of the surface, with the intent of showing that the coupling of resonators allows to tunnel elastic waves without creating straight channels. Resonators are separated by $( \pm 2, \pm 1)$ or $( \pm 1, \pm 2)$ lattice shifts, i.e. their absolute separation distance is fixed by the direction of the chain jumps from resonator to resonator. To verify the precision of the fabrication, an optical microscope (Leica model DM8000) is used to check the dimensions of the fabricated structures as shown in Fig. 1(d). The resonators along the chain are numbered in sequence. The source region is placed inside the phononic crystal. For frequencies inside the complete phononic band gap this ensures that the source region is isolated from the frame surrounding the phononic crystal structure. Evanescent coupling decreases very fast with distance, resulting effectively in near-neighbor coupling along the chain. Since the first resonator is the only one close to the source region, it is the only resonator that is significantly coupled with it.

Measurements of vibrations of the chain are conducted by using a laser Doppler vibrometer (Polytec Microsystem Analyzer MSA-500, equipped with analog displacement decoder model DD-300; 0.03-24 MHz frequency response with $50 \mathrm{~nm} / \mathrm{V}$ sensitivity). A periodic chirp is chosen as the source waveform in order to cover the frequency range of interest. The electric signal is transformed to a displacement vibration signal via the PZT transducer. The vertical, or out-of-plane, displacement signal is recorded by the vibrometer at the top surface of the sample.

All numerical simulations in this work are implemented using the finite element method in three dimensions (3D). The mechanical material parameters used for isotropic fused silica are mass density $\rho=2201 \mathrm{~kg} / \mathrm{m}^{3}$, Poisson's ratio $\nu=0.17$, and Young's modulus $E=72.5 \mathrm{GPa}$. A $3 \mathrm{D}$ finite element model was set to compare with vibration experiments. The mesh encompasses the full $20 \times 18$ phononic crystal of cross holes, the chain of resonators, and the source. To minimize wave reflections at the lateral boundaries of the mesh, radiation boundary conditions are applied. To allow for a direct comparison with experiment, a time-harmonic and spatially random wave source of vertical polarization is applied at the source region $^{32}$. With this setting, all possible modes of vibration are excited as the frequency is swept though the band gap range. The frequency response function (FRF) can be recorded at any position along the sample surface. Dispersion relations and phononic band structures are obtained by solving an eigenvalue problem with periodic boundary conditions applied at the external boundaries of the unit cell or super-cell. The phononic crystal slab has a complete band gap extending from 1.5 to 4 $\mathrm{MHz}$. Material loss can be estimated with the $Q f$ factor (product of quality factor by operating frequency in Hertz). $Q f \approx 510^{12}$ for fused silica ${ }^{3}$. With the largest frequency considered being $4 \mathrm{MHz}$, we then have in principle $Q>1.2510^{6}$ for the material loss limit. Given our experimental resolution, such fine line width can not be resolved in practice. As a result material damping was not added to numerical simulations. Note, however, that radiation damping, resulting from the finite number of phononic crystal rows around the resonator chain, is taken into account through the use of radiation boundary 
(a)

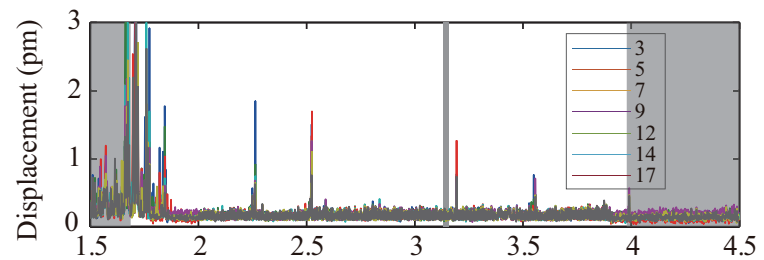

(b)

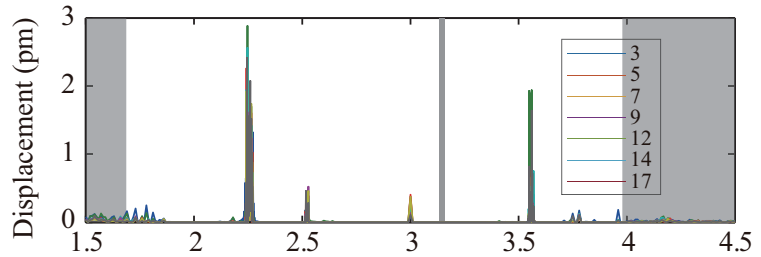

(c)

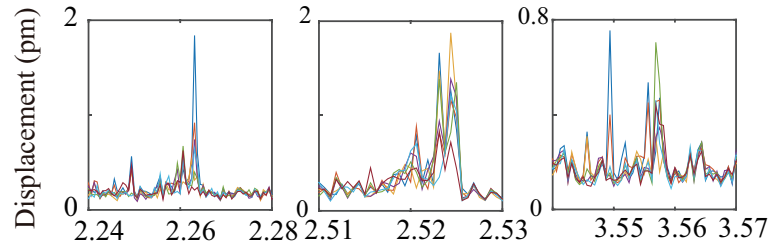

(d)

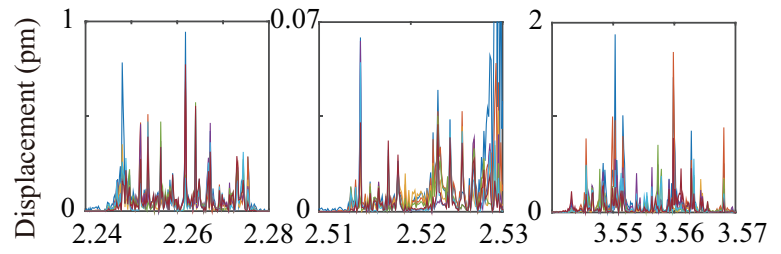

(e)

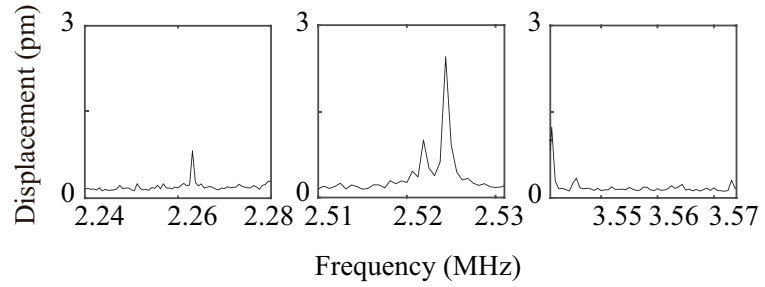

Figure 2. (a) Experimental and (b) numerical absolute vertical displacements at the position of the resonators. Each line color is for a particular resonator as labeled in Fig. 1(b). The complete band gap extends in the white region. (c)-(d) Zoomed views around selected frequencies. (e) Absolute experimental vertical displacements at the location of the source of vibrations are shown for comparison.

conditions.

\section{RESULTS}

The experimental measurement of the frequency response function (FRF) is presented in Fig. 2(a). The line colors represent the FRF measured at different resonators. The FRF shows a series of sharp resonances within the complete phononic band gap, corresponding to vibration modes of the sample. We consider particularly in the following the frequency regions around 2.26 $\mathrm{MHz}, 2.53 \mathrm{MHz}$, and $3.55 \mathrm{MHz}$, where the clearest signals are observed. When looking closer, it is observed that each main response actually subdivides in a series of closely spaced sharp resonances, as shown in Fig. 2(c). The number of sub-peaks is of the order of 18 in each case, i.e. a number related to the number of coupled resonators in the chain. A similar observation was made previously regarding the channeled transmission spectrum of sonic crystal waveguides ${ }^{33}$. The maximum amplitude of the vertical displacement of each resonance peak varies notably, indicating that vibration modes are variously matched to the excitation source. The vertical motion at the source location is shown in Fig. 2(e) for comparison. It can be observed that the resonance peaks measured at the location of the source do not match those measured inside the chain of resonators. This is expected, since at the location of the source thickness resonances ${ }^{34}$ of the fused silica plate should be excited and these have a different dispersion spectrum compared to the resonators. Interestingly, even when the source region vibrates very faintly, the vibrations of the chain of resonators can still be excited until the very last resonator.

Experimental results can be compared with the numerical results shown in Fig. 2(b) and (d). In the three frequency ranges extending around $2.26 \mathrm{MHz}, 2.53 \mathrm{MHz}$, and $3.55 \mathrm{MHz}$, series of sharp resonances are obtained with a frequency spacing in fair agreement with experiment. Note that the experimental frequency resolution is limited to $1.56 \mathrm{kHz}$, due to a limitation on the number of sample points, while numerical computations are presented with a resolution of $0.2 \mathrm{kHz}$ to ensure that each peak is resolved. Generally, numerical and experimental results agree fairly well, except for frequencies around 3 $\mathrm{MHz}$, where a significant FRF is only observed in the numerical simulation. We attribute this small discrepancy to the actual experimental environment that differs from the numerical excitation source: the experimental excitation is not truly spatially random as is assumed in the numerical simulation. Furthermore, it may be that the excitated vibrations have too small amplitude to be observed over the experimental noise floor.

Figure 3 displays the displacement maps observed at selected frequencies in both experiment and simulation. Animations of the vertical displacement distribution at frequencies $2.2625 \mathrm{MHz}, 2.5234 \mathrm{MHz}$, and 3.5578 $\mathrm{MHz}$ are further shown in Videos 1, 2, and 3. It is verified that all resonators oscillate collectively at the same frequency. In all cases, vibrations are mainly concentrated at the four corners of the resonators. A clear modal shape repeats for every resonator, in correspondence with the initial vibration mode of the resonator. The collective vibrations are for the whole chain of resonators, without apparent spatial attenuation along the chain, and are only limited by the experimental size of the array. 

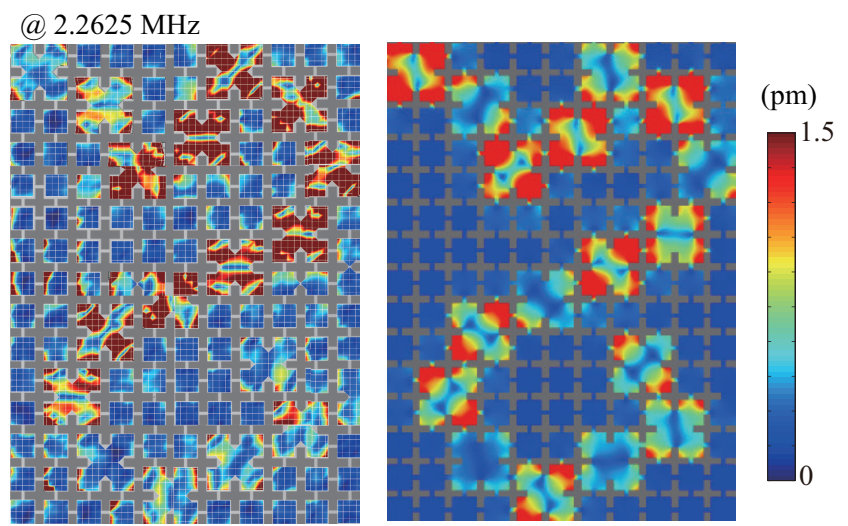

(a) $2.5234 \mathrm{MHz}$
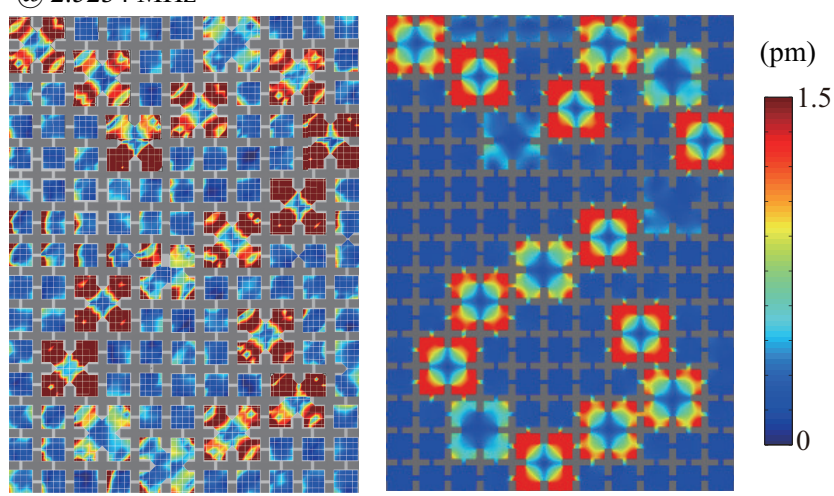

(a) $3.5578 \mathrm{MHz}$

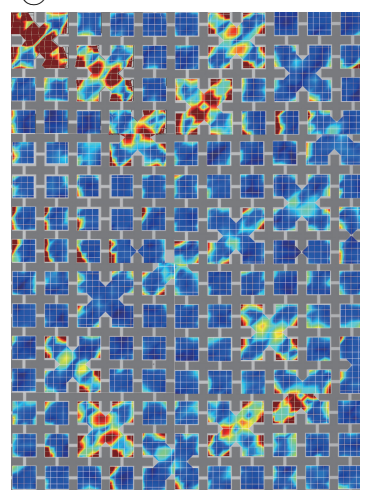

(a)

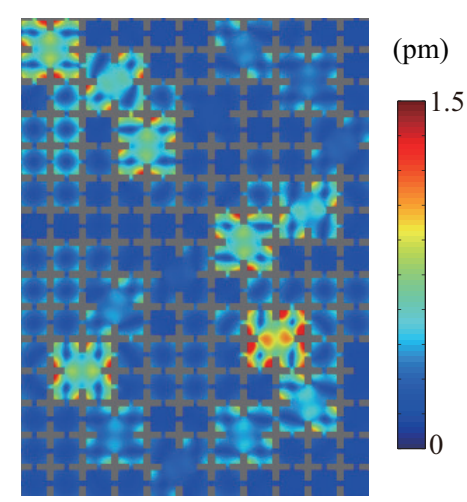

(b)

Figure 3. Vertical displacement maps at frequencies 2.2625 $\mathrm{MHz}, 2.5234 \mathrm{MHz}$, and $3.5578 \mathrm{MHz}$, in experiment (a) and numerical simulation (b), respectively. The color scale is for the amplitude of the vertical displacement from 0 (blue) to maximum (red). Numerical values are globally scaled to experimental values.

\section{MODEL OF THE PHONONIC POLYMER}

\section{A. Discrete sequence of eigenfrequencies}

The chain of coupled resonators forming a phononic polymer can be analyzed with various discrete models. Hamiltonian models have been proposed, especially in the context of topological phononics ${ }^{35-37}$. Following the

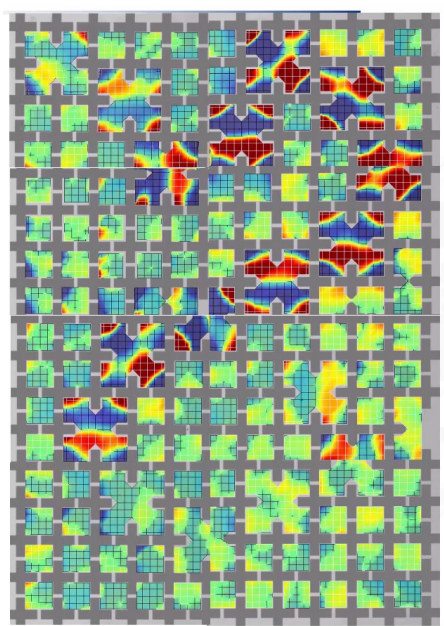

Video 1. Animation of the experimental vertical displacement distribution at frequency $2.2625 \mathrm{MHz}$.

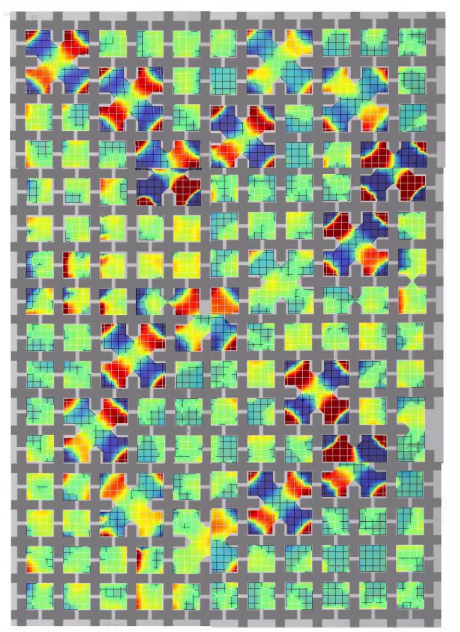

Video 2. Animation of the experimental vertical displacement distribution at frequency $2.5234 \mathrm{MHz}$.

Su-Schrieffer-Heeger (SSH) model of polyacetylene ${ }^{38}$, a coupling Hamiltonian can be constructed for eigenstates combining the degrees of freedom describing each resonator. Solving for the Schrödinger equation with the coupling Hamiltonian gives the sequence of eigenfrequencies. Such a technique parallels the dynamical matrix approach used to describe the dispersion of phonons in atomic lattices. Here, we consider a classical version of such quantum techniques ${ }^{39}$ where the state of the phononic polymer is described by one macroscopic degree of freedom per resonator, $U_{n}$. All resonators are identical except for a spatial shift in the crystal and have the same isolated resonance frequency $\omega_{0}$. By isolated resonator, we mean a resonator embedded in the infinite crystal and placed very far from any other resonator.

The dynamical equation for coupled resonators is writ- 


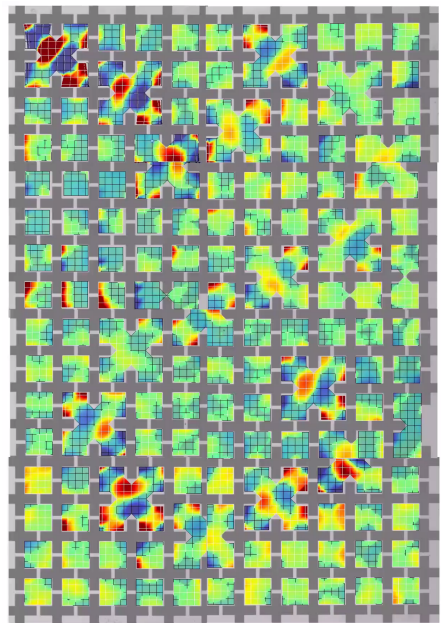

Video 3. Animation of the experimental vertical displacement distribution at frequency $3.5578 \mathrm{MHz}$.

ten

$$
-\ddot{U}_{m}=\sum_{n=1}^{N} D(m, n) U_{n},
$$

with a symmetric dynamical matrix $D(m, n)$ such that $D(m, m)=\omega_{0}^{2}$. The symmetry of the dynamical matrix is a consequence of reciprocity. Considering timeharmonic vibrations at a frequency $\omega$, the dynamical equation leads to eigenfrequencies that are eigenvalues of the dynamical matrix:

$$
\omega^{2} U_{m}=\sum_{n=1}^{N} D(m, n) U_{n} .
$$

As a result, there are exactly $N$ eigenstates of vibration of the phononic polymer. The symmetry of the dynamical matrix implies that the eigenfrequencies $\left(\omega_{i}, i=1 \cdots N\right)$ are real. This is the most general form we can obtain without making further simplifying assumptions.

If the resonators are distributed evenly along the chain, it can be assumed that the dynamical matrix is banded, i.e. $D(m, n)=\gamma_{|m-n|}$, reflecting the idea of a translationally invariant chain. The coefficients $\gamma_{|m-n|}$ account for nearest-neighbor coupling. This particular assumption was made for coupled-resonator waveguides formed of an infinite chain of coupled resonators ${ }^{40}$. The dispersion relation for Bloch waves of the infinite periodic chain was then obtained as $^{39}$

$$
\omega^{2}=2 \sum_{m=0}^{\infty} \gamma_{m} \cos (k m \Delta) .
$$

We stress that this formula applies to the phononic polymer only in the limit $N \rightarrow \infty$.

If we further assume that only nearest-neighbor cou- pling occurs, then the dynamical matrix simplifies to

$$
D=\left(\begin{array}{ccccc}
\omega_{0}^{2} & \gamma & 0 & \cdots & 0 \\
\gamma & \ddots & \ddots & \ddots & \vdots \\
0 & \ddots & \ddots & \ddots & 0 \\
\vdots & \ddots & \ddots & \ddots & \gamma \\
0 & \ldots & 0 & \gamma & \omega_{0}^{2}
\end{array}\right)
$$

For this particular case, the eigenfrequencies can be obtained analytically and are

$$
\omega_{N, m}^{2}=\omega_{0}^{2}+2 \gamma \cos \left(\frac{\pi m}{N+1}\right), m=1 \cdots N .
$$

This result is derived in the Appendix, where it is shown that a sequence of orthogonal polynomials is formed, whose zeros are the eigenfrequencies. As a consequence, the eigenfrequencies of polymer chains with increasing number of resonators are interleaved. It is further obtained that they distribute on the continuous dispersion relation for the infinite polymer

$$
\omega^{2}=\omega_{0}^{2}+2 \gamma \cos (k \Delta)
$$

where $\Delta$ is the period of the chain and with the wavenumber being sampled according to $k \Delta=\frac{\pi m}{N+1}, m=1 \cdots N$. This condition is equivalent to the phase-matching condition introduced to describe the channeled spectrum of the transmission of phononic crystal waveguides ${ }^{33}$. As a generalization, we infer that if the dispersion relation $\omega^{2}(k \Delta)$ is known, then the resonance frequencies will be given by $\omega^{2}\left(\frac{\pi m}{N+1}\right), m=1 \cdots N$. This distribution explains the sequence of sub-peaks in Fig. 2 around each initial defect resonance.

\section{B. Comparison to coupled-resonator waveguide}

Direct comparison of the discrete phononic polymer model with a coupled-resonator waveguide is not strictly possible since the former is aperiodic while the latter is periodic. Anyway, in order to enable comparison to some extent, we consider a simplified periodic version of the phononic polymer, as depicted by the super-cell in Fig. 4(a). Compared to the actual chain of coupled resonators considered in experiments, the coupled-resonator waveguide of Fig. 4(a) implements a sequence of lattice translations $(2,-1)$ then $(2,+1)$ so that the spatial period along axis $x$ is $4 a$ (four crosses along axis $x$ in the super-cell). The coupled-resonator waveguide thus defined could be obtained by a continuous deformation of the chain of resonators.

Figure 4(b) shows the phononic band structure for the coupled-resonator waveguide whose super-cell is presented in Figure 4(a). The color bar measures the polarization amount of the vertical component of displacement. Compared to the phononic band structure of the 


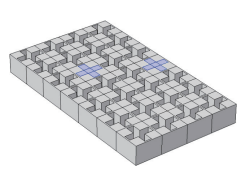

(a)

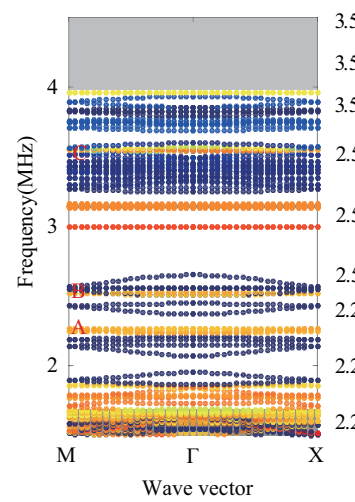

(b)

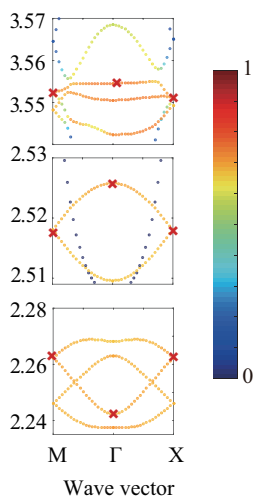

(c)

Figure 4. Dispersion of an equivalent coupled-resonator elastic waveguide. Supercell (a), band structure (b), and zoomed band structures (c) around 2.26 MHz, 2.52 MHz, and 3.55 $\mathrm{MHz}$, corresponding to point $\mathrm{A}, \mathrm{B}$ and $\mathrm{C}$, respectively.

Blue (red) corresponds to zero (maximum) amplitude for the out-of-plane displacement field. The gray areas indicate the passing frequency ranges.

perfect crystal, additional bands appear inside the complete band gap. Figure 4(c) shows close-up views of the band structure around $2.26 \mathrm{MHz}, 2.52 \mathrm{MHz}$, and 3.555 $\mathrm{MHz}$, corresponding to labels $\mathrm{A}, \mathrm{B}$, and $\mathrm{C}$ inserted in panel (a). As a note, the $4 a$ period causes spurious foldings at the $\mathrm{X}$ and $\mathrm{M}$ points of the first Brillouin zone, since two periods of the chain are actually included in the super-cell. As a result, there are 2 bands in spectral ranges $\mathrm{A}$ and $\mathrm{B}$, and presumably more in spectral range $\mathrm{C}$. These bands have different polarization contents and thus couple differently with the source of vibrations. In particular, the mostly vertically polarized band in spectral range B has the cosine shape of Eq. (6), in correspondence with the single coupling coefficient of the dynamical matrix in Eq. (4). The other bands do not have this simple cosine shape and more coupling coefficients should be included to describe them. Significantly, all bands extend almost symmetrically toward the $\mathrm{X}$ and the $\mathrm{M}$ point, indicating that the coupling coefficients are here mostly independent of the direction of the coupling, as we have implicitly assumed in the discrete model.

Eigenmodes at the high symmetry points of the Brillouin zone on three selected bands of spectral ranges $\mathrm{A}$, $\mathrm{B}$, and $\mathrm{C}$ are illustrated in Fig. 5. It can be seen that the eigenmodes in spectral range $\mathrm{B}$ are identical for all high symmetry points. We further verified that they are identical with the defect mode of the isolated resonator. More significantly, the modal shapes are clearly identical with those of Fig. 3, supporting the conclusion that the collective vibrations of the chain result from evanescent coupling of the individual resonators. In spectral range $\mathrm{A}$, the situation is a bit more intricate, as two degenerate isolated defect modes co-exist. They form two separate bands when unfolded, with a dispersion involving more than one Fourier harmonic, i.e. corresponding to the

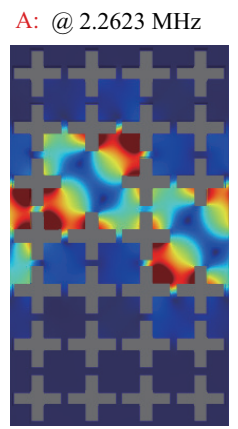

B: @ $2.5180 \mathrm{MHz}$

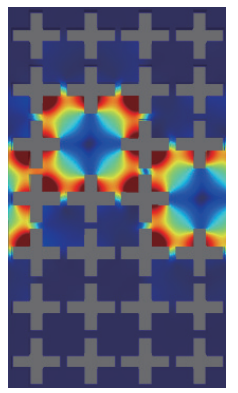

C: @ $3.5521 \mathrm{MHz}$

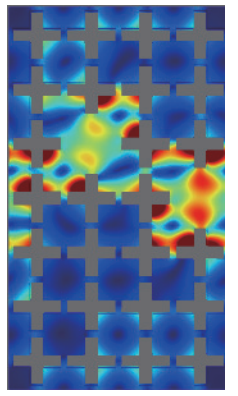

M point

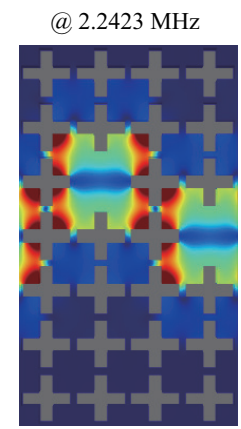

(a) $2.5258 \mathrm{MHz}$

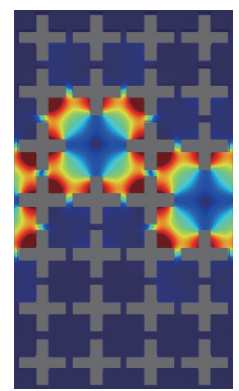

(a) $3.5546 \mathrm{MHz}$

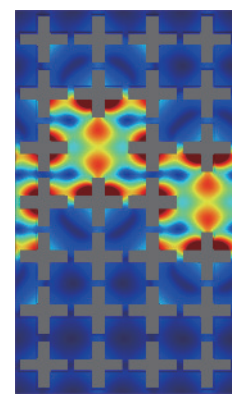

$\Gamma$ point

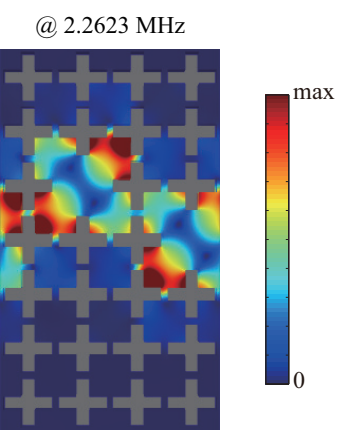

(a) $2.5180 \mathrm{MHz}$

(a) $3.5512 \mathrm{MHz}$

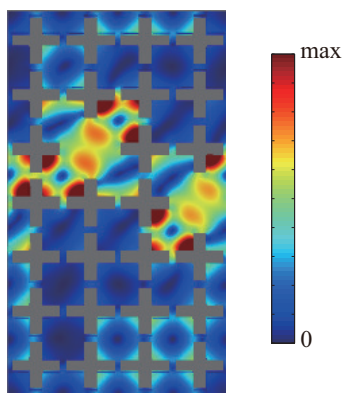

$\mathrm{X}$ point

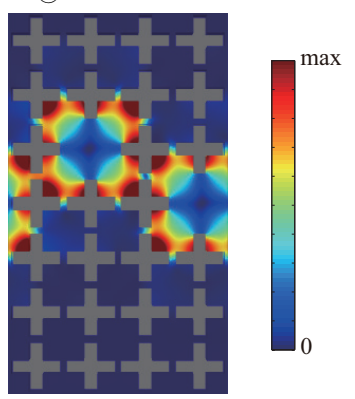

Figure 5. Eigenmodes of the supercell shown at the M point, $\Gamma$ point and $\mathrm{X}$ point of the first Brillouin zone at the passing bands corresponding to A, B, and C labeled in Fig. 4. Blue (red) corresponds to zero (maximum) amplitude for the displacement field.

general form (3) rather to the simpler (6). In spectral range $\mathrm{C}$, the bands do not have a pure out-of-plane vibration character, and the discrete model of the phononic polymer should be enriched with up to three degrees of freedom per resonator, which we leave as a perspective.

As remarked in Section II, the band appearing around $3 \mathrm{MHz}$ in the numerical simulation is not observed in the experiment. For completeness, however, we discuss briefly its characteristics. The eigenmodes at the $\mathrm{M}$ point, the $\Gamma$ point and the $\mathrm{X}$ point of the first Brillouin zone are shown in Fig. 6(a). These eigenmodes are identical for all three high symmetry points. Fig. 6(b) further displays the numerical displacement distribution in the chain of resonators at $3 \mathrm{MHz}$. The band in Fig. 6(c) has a cosine shape similar to the out-of-plane band in range B, indicating the dominance of nearest-neighbor coupling. Overall, the vibration characteristics at $3 \mathrm{MHz}$ should 
(a)

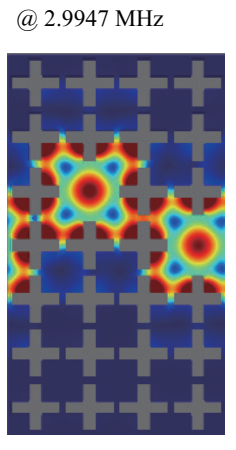

M point

(b)

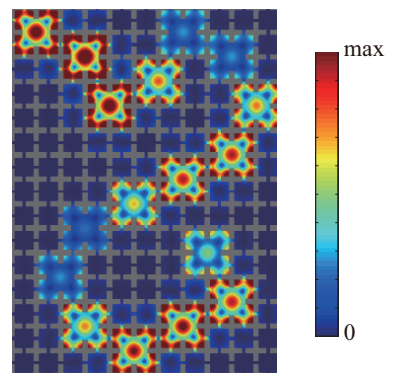

@ $2.9982 \mathrm{MHz}$

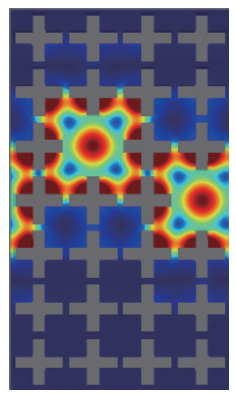

$\Gamma$ point (a) $2.9947 \mathrm{MHz}$

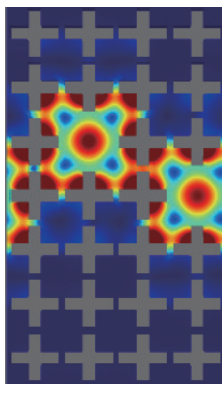

X point

(c)

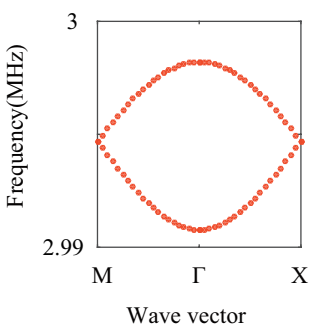

Figure 6. Characteristics of the band appearing around $3 \mathrm{MHz}$. (a) Eigenmodes are shown at the $M$ point, the $\Gamma$ point and the $\mathrm{X}$ point of the first Brillouin zone. (b) Numerical vertical displacement map at $2.9947 \mathrm{MHz}$ for the chain of resonators. (c) Band structure of the equivalent coupledresonator elastic waveguide.

be mostly similar to those at $2.5 \mathrm{MHz}$. Finally, we compare in Appendix B the acoustic transmission rate between the phononic polymer and conventional phononic waveguides with a variable number of bends.

\section{CONCLUSION}

In this paper, coupled elastic vibrations of a chain of coupled resonators in a square lattice phononic crystal slab have been studied. Coupling of resonators is evanescent within the wide complete band gap obtained with cross holes, resulting in nearest-neighbor coupling. We designed the chain of resonators by omitting the etching of selected holes. Measurements show that collective vibrations of the evanescently coupled resonators are efficiently excited. We imaged the modal vibrations over the surface and observed that the resonators oscillate collectively at the same frequency. Significantly, collective oscillations survive although the chain is aperiodic and does not form a classical phononic crystal waveguide. The number of resonances is as a rule equal to the number of resonators in the chain, in a first approximation. The evanescent coupling mechanism we consider is indeed reminescent of coupled-resonator waveguides. The main difference is that we do not require periodicity in the plane. This relaxed assumption allows us to design chains of resonators with a rather arbitrary shape.

This work provides a foreground for the consideration of phononic polymers, where resonators with equal free resonance frequency are arranged in an arbitrary chain and coupled evanescently. Active or even smart manipulation of localized resonators is thus expected. Such phononic polymers could be used for fusion sensing purposes, with each resonator functionalized to the same or different analytes. It is expected that the collective vibrations should be highly sensitive to a local change in one of the resonators. Phononic polymers could also be employed in optomechanics, using light forces such as radiation pressure to excite and detect collective resonances without any mechanical contact. Furthermore, by tuning the coupling coefficients between resonators, it should be possible to implement topological phononics models inspired for instance by the Su-Schrieffer-Heeger ( $\mathrm{SSH})$ model of the molecule of polyacetylene.

The fabrication process we employed relies on the locally-selective etching of fused silica. Other microfabrication techniques could obviously be alternatively selected, in view of different material platforms or to adjust the frequency range of the ultrasonic resonances.

\section{ACKNOWLEDGMENTS}

This work was supported by the French RENATECH network and its FEMTO-ST technological facility. Financial support by the National Natural Science Foundation of China (11702017 and 11532001) is gratefully acknowledged. V.L. acknowledges financial support by the EIPHI Graduate School (ANR-17-EURE-0002). T.T.W. acknowledges a scholarship provided by the China Scholarship Council (No. 201707090056).

\section{Appendix A: Scalar model of a chain of equally coupled resonators}

Let us consider of finite chain of $N$ discrete resonators described by Eq. (4). The dynamical matrix has the form $D_{N}=\omega_{0} I_{N}+\gamma C_{N}$ where $I_{N}$ is the identity matrix of size $N$ and

$$
C_{N}=\left(\begin{array}{ccccc}
0 & 1 & 0 & \cdots & 0 \\
1 & \ddots & \ddots & \ddots & \vdots \\
0 & \ddots & \ddots & \ddots & 0 \\
\vdots & \ddots & \ddots & \ddots & 1 \\
0 & \ldots & 0 & 1 & 0
\end{array}\right)
$$

The eigenvalues of the latter matrix are roots of the characteristic polynomial $P_{N}(X=\lambda)=\operatorname{det}\left(C_{N}-\lambda I_{N}\right)$. It is easy to check that the set of polynomials $P_{N}$ thus defined satisfy the recurrence relation

$$
P_{N+2}+X P_{N+1}+P_{N}=0, N \geq 0,
$$




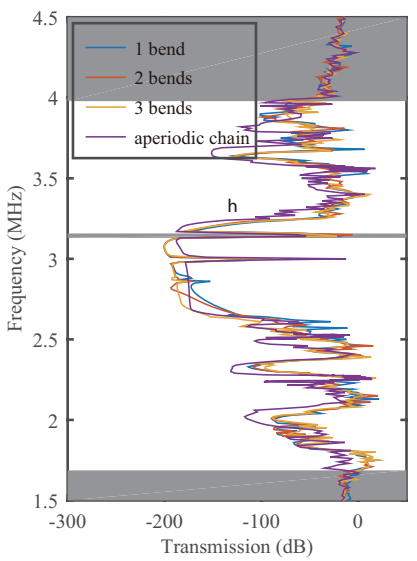

(a)

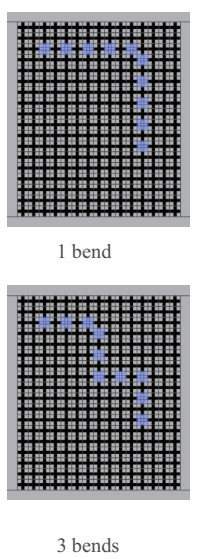

(b)

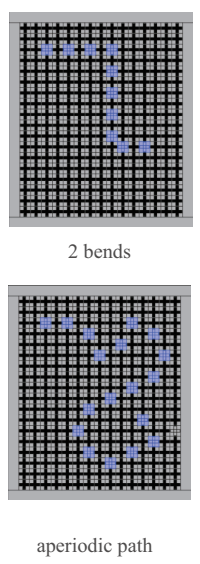

)
Figure 7. (a) Transmission spectra for three circuits with a different number of bends and for the aperiodic chain. The circuits are shown in (b).

with the first two polynomials being $P_{0}=1$ and $P_{1}=$ $-X$. The next polynomials are $P_{2}=X^{2}-1, P_{3}=$ $-X^{3}+2 X, P_{4}=X^{4}-3 X^{2}+1$, and so on.

The existence of the recurrence relation (A2) implies that the set of the real polynomials $P_{N}$ is orthogonal. As a consequence, their roots are all real and are interleaved. The recurrence relation actually resembles the one for Tchebychev polynomials, $T_{N+2}-2 X T_{N+1}+T_{N}=0$. Tchebychev polynomials are characterized by the relation $T_{N}(\cos \theta)=\cos (N \theta)$ for $0 \leq \theta \leq \pi$, but are not the only set of orthogonal polynomials determined by this recurrence relation. In particular, their derivatives $U_{N}=\frac{1}{N} T_{N}^{\prime}$ also do. Those polynomials satisfy $U_{N}(\cos \theta)=\sin (N \theta) / \sin (\theta)$ and are $U_{1}=1, U_{2}=2 X$, $U_{3}=4 X^{2}-1, U_{4}=8 X^{3}-4 X$ and so on. It is easily checked that $P_{N}(X)=U_{N+1}(-2 X)$. The zeros of
$U_{N+1}$ are given by condition $\sin ((N+1) \theta) / \sin (\theta)=0$, or $\theta_{m}=\frac{m \pi}{N+1}, m=1 \cdots N$. As a result, the zeros of $P_{N}$ are $\lambda_{m}=-2 \cos \left(\theta_{m}\right)=-2 \cos \left(\frac{m \pi}{N+1}\right), m=1 \cdots N$ and are all in interval $[-2,2]$.

\section{Appendix B: Waveguide transmission}

The sample we prepared was designed to observe the collective vibrations of the chain of resonators, but not specifically to demonstrate waveguiding. In order to obtain high transmission, it would be necessary to take care of the coupling of the source with the entrance of the waveguide, but also to reduce the reflection coefficient at the exit of the chain considered as a waveguide.

To compare the acoustic transmission rate between the phononic polymer and conventional phononic waveguides, we designed three additional and more traditional coupled-resonator waveguides with a different number of bends. Figure 7 shows the simulated transmission spectra. We calculated the transmission rate by

$$
T(f a)=\frac{\int_{S_{r}} U \mathrm{~d} s}{\int_{S_{l}} U_{0} \mathrm{~d} s}
$$

where $U$ is the total displacement at the exit and $U_{0}$ is the total displacement at the entrance of the waveguide. The main observation that we make is that transmission spectra are similar for all waveguides and for the phononic polymer, though the details of the spectra (separation between resonances) depend on the total length of each chain. Compared to conventional coupledresonator phononic waveguides, a distinctive advantage of the phononic polymer is that guided waves can propagate along a rather arbitrary path and not only along principal directions of the crystal.
* wangyanfeng@bjtu.edu.cn

$\dagger$ vincent.laude@femto-st.fr

1 M. S. Kushwaha, P. Halevi, L. Dobrzyński, and B. DjafariRouhani, "Acoustic band structure of periodic elastic composites," Phys. Rev. Lett. 71, 2022 (1993).

2 M. M. Sigalas and E. N. Economou, "Band structure of elastic waves in two dimensional systems," Solid State Commun. 86, 141 (1993).

3 V. Laude, Phononic crystals: artificial crystals for sonic, acoustic, and elastic waves (de Gruyter, Berlin, 2015).

${ }^{4}$ V. Laude, Y. Achaoui, S. Benchabane, and A. Khelif, "Evanescent Bloch waves and the complex band structure of phononic crystals," Phys. Rev. B 80, 092301 (2009).

5 J. S. Jensen, "Phononic band gaps and vibrations in oneand two-dimensional mass-spring structures," J. Sound Vib. 266, 1053-1078 (2003).

${ }^{6}$ X. An, H. Fan, and C. Zhang, "Elastic wave and vibration bandgaps in two-dimensional acoustic metamaterials with resonators and disorders," Wave Motion 80, 69-81 (2018).

7 T.-T. Wang, Y.-F. Wang, Y.-S. Wang, and V. Laude, "Evanescent-wave tuning of a locally resonant sonic crystal," Appl. Phys. Lett. 113, 231901 (2018).

8 Y.-F. Wang, V. Laude, and Y.-S. Wang, "Coupling of evanescent and propagating guided modes in locally resonant phononic crystals," J. Phys. D: Appl. Phys. 47, 475502 (2014).

9 M. Torres, F. R. Montero de Espinosa, D. García-Pablos, and N. García, "Sonic band gaps in finite elastic media: surface states and localization phenomena in linear and point defects," Phys. Rev. Lett. 82, 3054 (1999).

10 Y. Pennec, J. O. Vasseur, B. Djafari-Rouhani, L. Dobrzyński, and P. A. Deymier, "Two-dimensional phononic crystals: Examples and applications," Surf. Sci. Rep. 65, 229-291 (2010). 
11 Y. Akahane, M. Mochizuki, T. Asano, Y. Tanaka, and S. Noda, "Design of a channel drop filter by using a donortype cavity with high-quality factor in a two-dimensional photonic crystal slab," Appl. Phys. Lett. 82, 1341-1343 (2003).

12 T.-T. Wang, Y.-F. Wang, Y.-S. Wang, and V. Laude, "Tunable fluid-filled phononic metastrip," Appl. Phys. Lett. 111, 041906 (2017).

13 Y. Jin, N. Fernez, Y. Pennec, B. Bonello, R. P. Moiseyenko, S. Hémon, Y. Pan, and B. Djafari-Rouhani, "Tunable waveguide and cavity in a phononic crystal plate by controlling whispering-gallery modes in hollow pillars," Phys. Rev. B 93, 054109 (2016).

14 A. Yariv, Y. Xu, R. K. Lee, and A. Scherer, "Coupledresonator optical waveguide: a proposal and analysis," Opt. Lett. 24, 711-713 (1999).

15 M. Notomi, E. Kuramochi, and T. Tanabe, "Large-scale arrays of ultrahigh-Q coupled nanocavities," Nat. Photon. 2, 741 (2008).

16 T. Baba, "Slow light in photonic crystals," Nat. Photon. 2, 465 (2008).

17 L. Xia, F.and Sekaric and Y. Vlasov, "Ultracompact optical buffers on a silicon chip," Nat. Photon. 1, 65 (2007).

18 J. Wang, Z. Yao, T. Lei, and A. W. Poon, "Silicon coupledresonator optical-waveguide-based biosensors using lightscattering pattern recognition with pixelized mode-fieldintensity distributions," Sci. Rep. 4, 7528 (2014).

19 C. R. Otey, M. L. Povinelli, and S. Fan, "Completely capturing light pulses in a few dynamically tuned microcavities," J. Lightwave Technol. 26, 3784-3793 (2008).

${ }^{20}$ R. F. Oulton, V. J. Sorger, D. A. Genov, D. F. P. Pile, and X. Zhang, "A hybrid plasmonic waveguide for subwavelength confinement and long-range propagation," Nat. Photon. 2, 496 (2008).

21 A. Khelif, A. Choujaa, S. Benchabane, B. Djafari-Rouhani, and V. Laude, "Guiding and bending of acoustic waves in highly confined phononic crystal waveguides," Appl. Phys. Lett. 84, 4400-4402 (2004).

${ }^{22}$ D. Hatanaka, I. Mahboob, K. Onomitsu, and H. Yamaguchi, "Phonon waveguides for electromechanical circuits," Nat. Nanotechnol. 9, 520 (2014).

23 Y.-F. Wang, T.-T. Wang, J.-P. Liu, Y.-S. Wang, and V. Laude, "Guiding and splitting Lamb waves in coupledresonator elastic waveguides," Comp. Struct. 206, 588-593 (2018).

24 Y.-F. Wang, T.-T. Wang, Y.-S. Wang, and V. Laude, "Reconfigurable phononic-crystal circuits formed by coupled acoustoelastic resonators," Phys. Rev. Appl. 8, 014006 (2017).

25 I. E. Psarobas, N. Stefanou, and A. Modinos, "Phononic crystals with planar defects," Phys. Rev. B 62, 5536 (2000).

26 A. Khelif, A. Choujaa, B. Djafari-Rouhani, M. Wilm,
S. Ballandras, and V. Laude, "Trapping and guiding of acoustic waves by defect modes in a full-band-gap ultrasonic crystal," Phys. Rev. B 68, 214301 (2003).

27 Y. Pennec, B. Djafari-Rouhani, J. O. Vasseur, H. Larabi, A. Khelif, A. Choujaa, S. Benchabane, and V. Laude, "Acoustic channel drop tunneling in a phononic crystal," Appl. Phys. Lett. 87, 261912 (2005).

28 S. Benchabane, R. Salut, O. Gaiffe, V. Soumann, M. Addouche, V. Laude, and A. Khelif, "Surface-wave coupling to single phononic subwavelength resonators," Phys. Rev. Appl. 8, 034016 (2017).

29 L. Raguin, O. Gaiffe, R. Salut, J.-M. Cote, V. Soumann, V. Laude, A. Khelif, and S. Benchabane, "Coherent interplay between surface acoustic waves and coupled mechanical resonators: transition from plasmon-like to surface mediated coupling," Nat. Commun. 10, 4583 (2019).

30 Y. Bellouard, A. Said, M. Dugan, and P. Bado, "Fabrication of high-aspect ratio, micro-fluidic channels and tunnels using femtosecond laser pulses and chemical etching," Opt. Express 12, 2120-2129 (2004).

31 Audrey Champion and Yves Bellouard, "Direct volume variation measurements in fused silica specimens exposed to femtosecond laser," Opt. Mat. Express 2, 789-798 (2012).

32 V. Laude and M. E. Korotyaeva, "Stochastic excitation method for calculating the resolvent band structure of periodic media and waveguides," Phys. Rev. B 97, 224110 (2018).

33 Y.-F. Wang, T.-T. Wang, J.-W. Liang, Y.-S. Wang, and V. Laude, "Channeled spectrum in the transmission of phononic crystal waveguides," J. Sound Vib. 437, 410-421 (2018).

34 Thickness resonances are standing Lamb waves of the homogeneous plate, having a zero lateral wavenumber.

35 P. Wang, L. Lu, and K. Bertoldi, "Topological phononic crystals with one-way elastic edge waves," Phys. Rev. Lett. 115, 104302 (2015).

36 S. H. Mousavi, A. B. Khanikaev, and Z. Wang, "Topologically protected elastic waves in phononic metamaterials," Nat. Commun. 6, 8682 (2015).

37 R. Süsstrunk and S. D. Huber, "Observation of phononic helical edge states in a mechanical topological insulator," Science 349, 47-50 (2015).

38 W. P. Su, J. R. Schrieffer, and A. J. Heeger, "Solitons in polyacetylene," Phys. Rev. Lett. 42, 1698 (1979).

39 Jose Maria Escalante, Alejandro Martínez, and Vincent Laude, "Dispersion relation of coupled-resonator acoustic waveguides formed by defect cavities in a phononic crystal," J. Phys. D: Appl. Phys. 46, 475301 (2013).

40 For a finite chain of coupled resonators, there are in principle end effects that break the banded matrix assumption: resonators at either ends do not see the same environment as resonators in the middle of the chain. 